Proc. 13th International School on Theoretical Physics: Symmetry and Structural Properties of Condensed Matter

\title{
Non-Adiabatic Transformation of a Spin-Chain Geometry via Local Control
}

\author{
P.V. PYShKin ${ }^{a}$, E.YA. ShERMAN ${ }^{b, c, *}$ AND LiAn-Ao Wu ${ }^{c, d}$ \\ ${ }^{a}$ Institute for Solid State Physics and Optics, Wigner Research Centre, \\ Hungarian Academy of Sciences, P.O. Box 49, H-1525 Budapest, Hungary \\ ${ }^{b}$ Department of Physical Chemistry, The University of the Basque Country UPV/EHU, 48080 Bilbao, Spain \\ ${ }^{c}$ IKERBASQUE, Basque Foundation for Science, 48011 Bilbao, Spain \\ ${ }^{d}$ Department of Theoretical Physics and History of Science, \\ The University of the Basque Country UPV/EHU, 48080 Bilbao, Spain
}

\begin{abstract}
We consider transformation from a closed to an open spin chain and vice versa produced by changing single link strength in a pair of neighboring spins. We show that in the non-adiabatic time domain fidelity of such a process can be increased by proper choosing of the control function for spin-spin exchange coupling. We obtain this function for an antiferromagnetic quantum Ising chain and present heuristic reasons restricting possible timedependences of Hamiltonians applied for a high-fidelity control.
\end{abstract}

DOI: 10.12693/APhysPolA.135.1198

PACS/topics: quantum control, spin chains, adiabatic processes

\section{Introduction}

Precise control of complex quantum systems became important task in last decades due to the prospective of building a quantum computer [1] and experimental feasibility of such kind of control [2,3]. Entanglement is the main feature of quantum computing algorithms, thus producing and destroying entanglement between parts of a complex system [4] is a problem of importance and interest. Another type of problems, related to manipulation of entanglement, is a transformation of a complex system from a highly entangled ground state of the initial Hamiltonian $H_{i}$ to a demanded state, which is the ground state of another Hamiltonian $H_{f}$. These Hamiltonians $H_{i}$ and $H_{f}$ can be related to different geometry (or topology) of a complex system. We would like to emphasize that although our goal is the transformation of quantum states one into another, we make a correspondence between initial and final states and Hamiltonians. For examples, investigating a spin lattice, we can consider it as a graph with spins as vertices, spin-spin interactions as edges, and in such a case $H_{i}$ and $H_{f}$ correspond to different configurations of the edges. If $H_{i}\left(H_{f}\right)$ corresponds to a disconnected (connected) graph and $\left[H_{i}, H_{f}\right] \neq 0$, we have a modification in the system entanglement.

The task of a quantum ground state transformation can be solved via unitary process using adiabatic control when evolution is governed by a time dependent Hamiltonian $H(t)=a(t) H_{i}+b(t) H_{f}$, where $a(0)=1, a(T)=0$, $b(0)=0, b(T)=1$, and $T$ is an evolution time. This process is also the main recipe of adiabatic quantum com-

*corresponding author; e-mail: evgeny.sherman@ehu.es putation [5, 6] and quantum annealing (see, e.g. [7] for quantum spin systems). In most cases it is sufficient to set linear switching: $a(t)=1-t / T$ and $b(t)=t / T$ to get a demanded result. The problem here is that time $T$ must be sufficiently long to satisfy the adiabatic theorem conditions [8]. However, at a long evolution time the system undesired decoherence becomes possible, thus the challenge of adiabatic shortcuts appears. Some approaches to make adiabatic evolution shorter, such as counteradiabatic driving $[9,10]$, strength-pulsed (noise) control $[11,12]$ and others $[13,14]$ have been presented recently. A common feature of all these techniques, which is difficult to realize, is the need of control all parts of the quantum system. Note that in our task we do not need the quantum system to be in the instantaneous ground state of the intermediate Hamiltonian $H(t)(0<t<T)$ during the entire transformation process since we are interested only in the final state. In present paper we consider transformation from the ground state of a closed spin chain to the ground state of an open spin chain and show that it is possible to use local-only control of a special kind to increase the fidelity of the target state in the non-adiabatic time domain. Some general restrictions imposed on the control function will be presented and discussed.

\section{Results}

\subsection{The model}

The key element of our investigation is a timedependent antiferromagnetic quantum Ising Hamiltonian in a transverse field

$$
H(g)=\sum_{n=1}^{N-1} \sigma_{n}^{x} \sigma_{n+1}^{x}+g \sigma_{1}^{x} \sigma_{N}^{x}+B \sum_{n=1}^{N} \sigma_{n}^{z},
$$


where $\sigma^{x}, \sigma^{z}$ are the Pauli matrices, $B$ is an external magnetic field, $N$ is the number of spins in the chain, and $g$ is a time-dependent control parameter. We assume that quantum state of the chain is described by a vector $|\psi(t)\rangle$, and the initial state is $|\psi(0)\rangle=\left|\phi_{0}\right\rangle$, with $H(g=1)\left|\phi_{0}\right\rangle=\lambda_{0}\left|\phi_{0}\right\rangle$, and $\lambda_{0}$ being the minimum eigenvalue of $H(g=1)$. Note that the system evolves in time due to nonzero value of the commutator

$$
\begin{aligned}
& {\left[\sum_{n=1}^{N-1} \sigma_{n}^{x} \sigma_{n+1}^{x}+B \sum_{n=1}^{N} \sigma_{n}^{z}, g \sigma_{1}^{x} \sigma_{N}^{x}\right]=} \\
& 2 i g B\left(\sigma_{1}^{y} \sigma_{N}^{x}+\sigma_{1}^{x} \sigma_{N}^{y}\right) \neq 0,
\end{aligned}
$$

and the ability to control the process depends on the evolution of the product of spin components at the edge connecting the first and and the last spin.

The problem now is to make a "cut" of a chain, i.e. to drive a system into a state $|\psi(T)\rangle=\left|\chi_{0}\right\rangle$, where $\left|\chi_{0}\right\rangle$ is the ground state of the open chain Hamiltonian $H(g=0)$ (see Fig. 1). We can write the final state as a result of unitary evolution

$$
\begin{aligned}
& |\psi(T)\rangle=U(T)|\psi(0)\rangle, \\
& U(T)=\mathcal{T} \exp \left(-\mathrm{i} \int_{0}^{T} H\left(g\left(t^{\prime}\right)\right) \mathrm{d} t^{\prime}\right),
\end{aligned}
$$

where $\mathcal{T}$ stands for the time-ordering, and control function $g(t)=1$ for $t \leq 0$ and $g(t)=0$ for $t \geq T$.
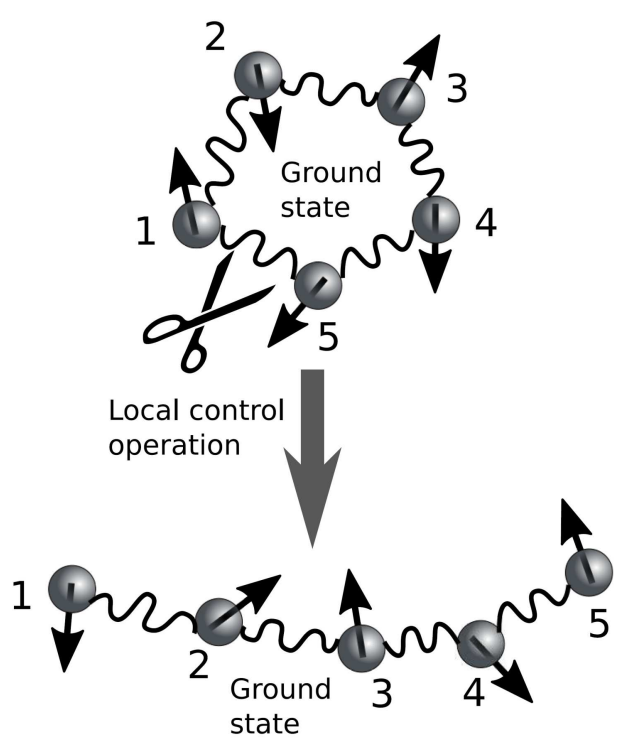

Fig. 1. Schematic illustration of transformation from a closed to an open spin chain.

We define the final target fidelity as follows:

$$
f_{T}=\left|\left\langle\chi_{0} \mid \psi(T)\right\rangle\right| \text {, }
$$

and consider it as a functional of the control function: $f_{T}=f_{T}[g(t)]$. The problem is to find the function $g(t)$ maximizing the target fidelity $f_{T}$ for fixed finitetime $T$.
In order to obtain a proper control function we make a two-parametric parametrization at the interval $t \in[0, T]$ :

$$
g(t)=1-\left(1+a_{1}+a_{2}\right) \frac{t}{T}+a_{1} \frac{t^{2}}{T^{2}}+a_{2} \frac{t^{3}}{T^{3}},
$$

and the target fidelity becomes a function of control parameters $f_{T}=f_{T}\left(a_{1}, a_{2}\right)$.

\subsection{The features of efficient control}

Before going further we emphasize that we restrict our consideration to the following condition:

$$
E_{0}(g) \neq E_{1}(g), \quad \forall g \in(0,1),
$$

where $E_{0}(g)\left(E_{1}(g)\right)$ is the instant ground (first excited) state energy of the Hamiltonian (1). If the condition (7) is valid then it is possible to make the adiabatic transformation with

$$
f_{T 0} \equiv f_{T}(0,0) \rightarrow 1, \quad T \rightarrow \infty .
$$

We can argue that for a large enough $T$ a linear decrease (8) is the optimal shape of the control. From the corresponding Schrödinger equation in the adiabatic frame we can see that probability of leaving the instantaneous eigenstate of $H(t)$ is proportional to the time derivative of Hamiltonian $|\partial H / \partial t| \propto|\partial g(t) / \partial t|$ (see e.g. textbook [15]). Thus, the adiabaticity condition can be written as $\partial H / \partial t \rightarrow 0$, and if one changes $g(t)$ for some fixed large $T$ from linear dependence into some other shape it necessarily leads to increase of $|\partial H / \partial t|$ (for some $t \in(0, T)$ ), and consequently the adiabaticity condition tends to be broken. Another way to see that the linear decrease is the optimal control in the adiabatic domain is the following reasoning. Let us take a large enough $T$ which corresponds to the adiabaticity regime for linear decrease, and periods $T^{\prime}$ with $T^{\prime}>T$ also satisfy adiabaticity condition. Now, let us modify the control function a little in two different ways (see Fig. 2):

(i) we start linear decrease not from $t=0$ but from $t=\delta t$, or

(ii) we finish linear decrease not at $t=T$ but at $t=T-\delta t$.

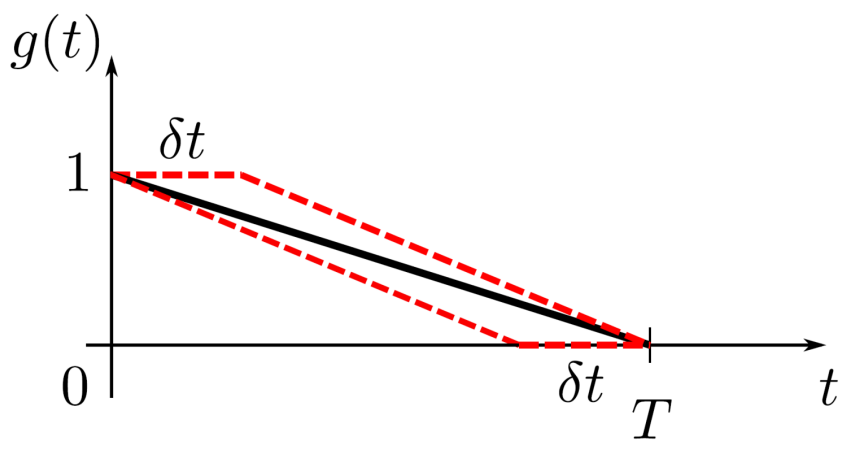

Fig. 2. Schematic illustration of the fact that linear dependence is the optimal one for near-adiabatic regime. Red dashed lines correspond to the modifications of the linear decrease which is shown by a bold solid line. Obviously, the modified control is not better for adiabaticity than the non-modified one. 
Both described modifications of the control function are nothing else than just decreasing the effective time $T \rightarrow T-\delta t$. The latter means that adiabaticity with modified control function will not be better than that for the linear shape, and by setting $\delta t \rightarrow 0$ we conclude that the linear decrease is the optimal shape for relatively large $T$. This conclusion also can be seen from the numerical results in Ref. [4].

Our conclusion (8) now will help us to understand the restrictions imposed on the control function $g(t)$ in a nonadiabatic time domain. We claim that any efficient control function must satisfy the following inequalities:

$$
\left.\frac{\mathrm{d} g(t)}{\mathrm{d} t}\right|_{t=0}<0,\left.\quad \frac{\mathrm{d} g(t)}{\mathrm{d} t}\right|_{t=T}<0 .
$$

The explanation of the restrictions (9) is the following. Let us assume that the optimal control function in the non-adiabatic domain does not satisfy (9). Now we slightly increase the time $T$ toward the adiabatic domain. As we have already noticed, the optimal $g(t)$ shape in this region is a linear decrease (8), thus, with the increase of $T$ the optimal shape of $g(t)$ must be continuously transformed into the linear shape. The latter means that there must be some intermediate values of $T$ when $\mathrm{d} g(t) /\left.\mathrm{d} t\right|_{t=0}=0$ and $\mathrm{d} g(t) /\left.\mathrm{d} t\right|_{t=T}=0$. However, the control cannot be efficient if the derivative of the control function is zero at the start or at the end of a time interval, because one can consider that there is no control applied at all for $t \ll T$ and $T-t \ll T$. Thus we obtain a contradiction with our initial statement that $(9)$ is not valid for optimal control, and thus we conclude that (9) must be valid.
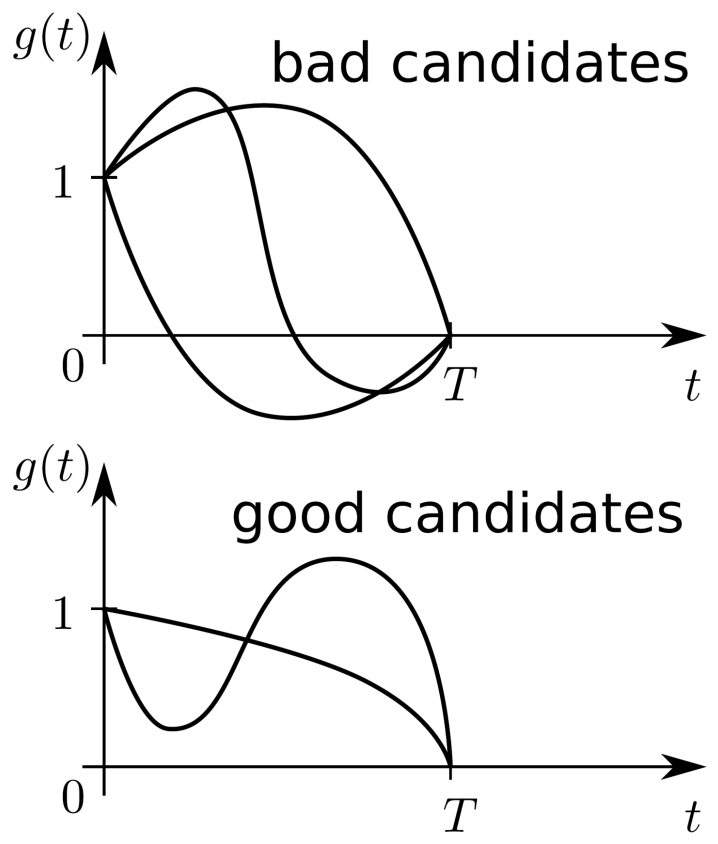

Fig. 3. Illustrative depiction of control functions not satisfying (10) (upper part) and satisfying (10) (lower part).
By substituting (6) into the two-parametric Ansatz (9) we get the following conditions:

$$
a_{2}>-1-a_{1}, \quad a_{2}<\frac{1-a_{1}}{2} .
$$

It is interesting to note that restrictions (10) do not depend on time $T$, being in some sense the universal ones, and connect together adiabatic and non-adiabatic time domains (even if we do not restrict the Ansatz in (6) to cubic terms). Obviously, the point $\left(a_{1}=0, a_{2}=0\right)$ satisfies (10). In Fig. 3 we show an illustrative example of control functions which are "bad candidates" (not satisfying (10)) and "good candidates" (satisfying (10)).

Although this analysis is not mathematically rigorous, it provides the solid basis for the understanding of the optimal control. Further numerical results presented below confirm our statement (9).

\subsection{Numerical results}

We use a numerical search in order to find the optimal values of $a_{1}$ and $a_{2}$ as can be made by using two different approaches such as:

(i) by brute-force evaluation of $f_{T}$ for all possible values of parameters (within some finite region and step size), in other words - building a landscape of fidelity; or

(ii) by using gradient search algorithms (for calculations in this paper we used the Broyden-Fletcher-GoldfarbShanno (BFGS) optimization method [16] which is builtin Python scientific packages).

Numerical calculations of time-ordered exponent (4) have been done by dividing the time interval $T$ to $M \gg 1$ pieces $\delta t=T / M$, and using approximation

$$
U(T) \approx \prod_{n=0}^{M-1} \exp (-\mathrm{i} \delta t H(g(\delta t(n+1 / 2)))) .
$$

Here we took $M=300$ and matrix exponents have been calculated via a built-in Python function.

Although the first approach needs large computational efforts, building the landscapes for searching of the optimized control functions can be done by parallel computing tools. In this paper we show that these two approaches give the same result. For numerical analysis we use the following Hamiltonian parameters: $N=5$ and $B=0.5$. With this choice we satisfy the level non-crossing condition (7) and in Fig. 4 present the dependence of the two lowest eigenvalues of the Hamiltonian (1).

In Fig. 5 we present the non-optimized fidelity $f_{T 0}$ and the $f_{T}$ obtained with optimized $g(t)$ found by the gradient search. We see that advantage of the using of optimized control appears in a wide range of non-adiabatic $T$.

Let us look into a landscape of the fidelity in the parameter space for $T=1$ as depicted in Fig. 6. White triangle corresponds to a linear decrease and white square corresponds to an effective control shape for this particular $T$. Area covered by the red dots corresponds to parameters which does not satisfy (10). As can be seen 


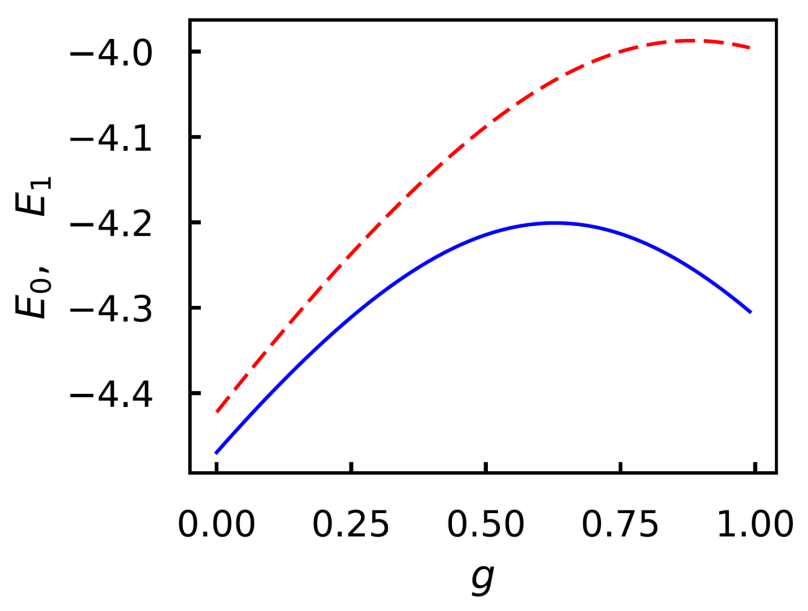

Fig. 4. The ground ( $E_{0}$, blue solid line) and the first excited $\left(E_{1}\right.$, red dashed line) state energy of the Hamiltonian (1) as a functions of $g$ parameter. Here $N=5$ and $B=0.5$.

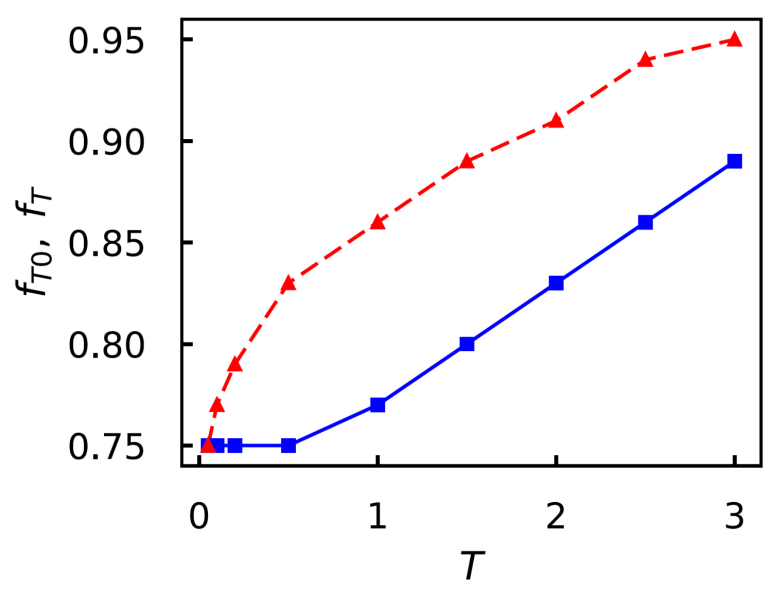

Fig. 5. Output fidelity of non-optimized (blue solid line) and optimized (red dashed line) transitions for cutting of a spin chain with $N=5$ and $B=0.5$.

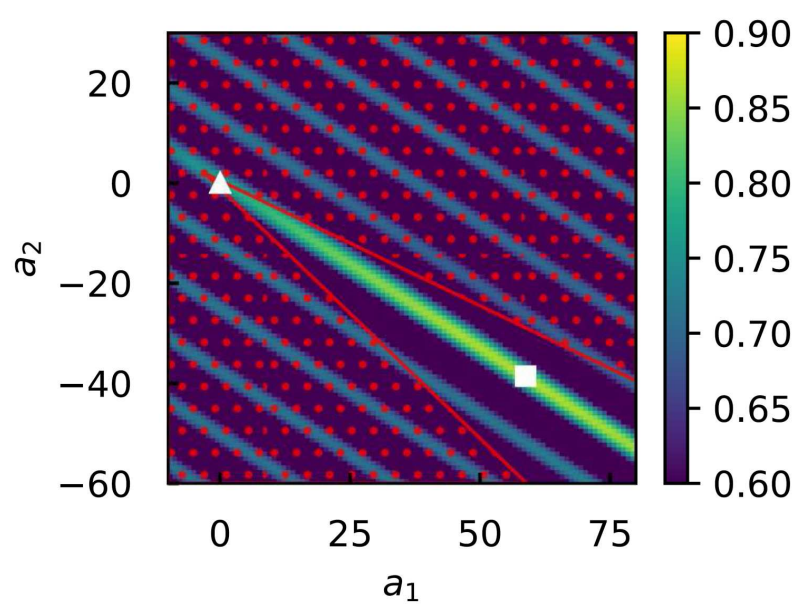

Fig. 6. Landscape of the target fidelity $f_{T}\left(a_{1}, a_{2}\right)$ for $T=1$. White triangle corresponds to the simple linear control and square corresponds to the maximal fidelity process. in this figure, there is a continuous "island" of a high fidelity connecting linear decrease (white triangle) and the optimal shape (white square). Thus, the numerical gradient search started from the linear shape is efficient in this situation.

Now let us return to our statement (8). Our numerical results confirm that the linear decrease is the optimal control in the adiabatic limit. In Fig. 7 we show the dependence of optimal values of parameters $a_{1}, a_{2}$ for different times $T$. One can see that all points are inside allowed by (10) area, and they converge to the linear decrease (depicted as triangle).

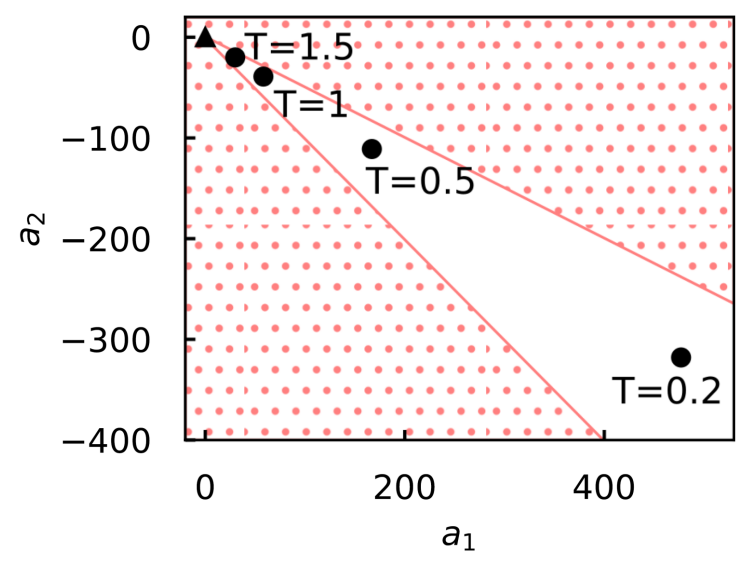

Fig. 7. Optimal values of parameters $a_{1}$ and $a_{2}$ for different $T$. All of them lie in the region determined by inequalities (10) (non-dotted area). Triangle is the adiabatic limit of control with the linear decrease.

In Fig. 8 we show the optimal shapes of control for some values of time $T$. As can be seen from Fig. 8, the calculated shapes correspond to a "good candidates" in Fig. 3.

\subsection{Pulsed control}

Inequalities (9) are written for a smooth control function. Now, let us assume that we apply for the control discontinuous rectangular pulses as

$$
g(t)=\sum_{n=1}^{K} b_{n}[\theta(t-(n-1) \Delta t)-\theta(t-n \Delta t)],
$$

where we have $K$ pulses with the duration $\Delta t=T / K$, amplitude of $n$-th pulse is $b_{n}$, and $\theta(t)$ is the Heaviside step function. In such a case by doing the same reasoning (for $K \gg 1$ ) as above we arrive at the following restrictions:

$$
b_{1}<1, \quad b_{K}>0 .
$$

However, we can see that restrictions (13) are valid even for small number of pulses. In Fig. 9 we show the landscape of fidelity for pulse control (12) for the case $K=2$. Instead of comparing optimal parameters with the simple linear shape as in Fig. 6, we assume "quasi-linear" step-like decrease with $b_{1}=2 / 3$ and $b_{2}=1 / 3$ as a nonoptimized control function and as a starting point for the numerical gradient search. 

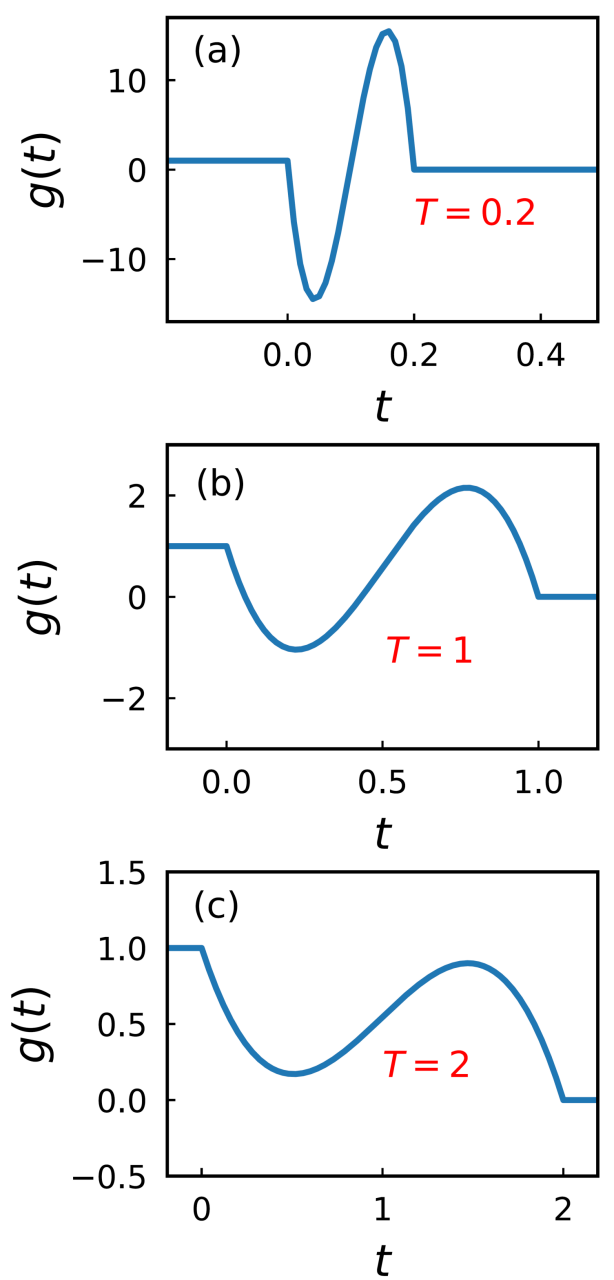

Fig. 8. Optimal control shapes for different $T$. All of them correspond to the "good candidates" in Fig. 3.

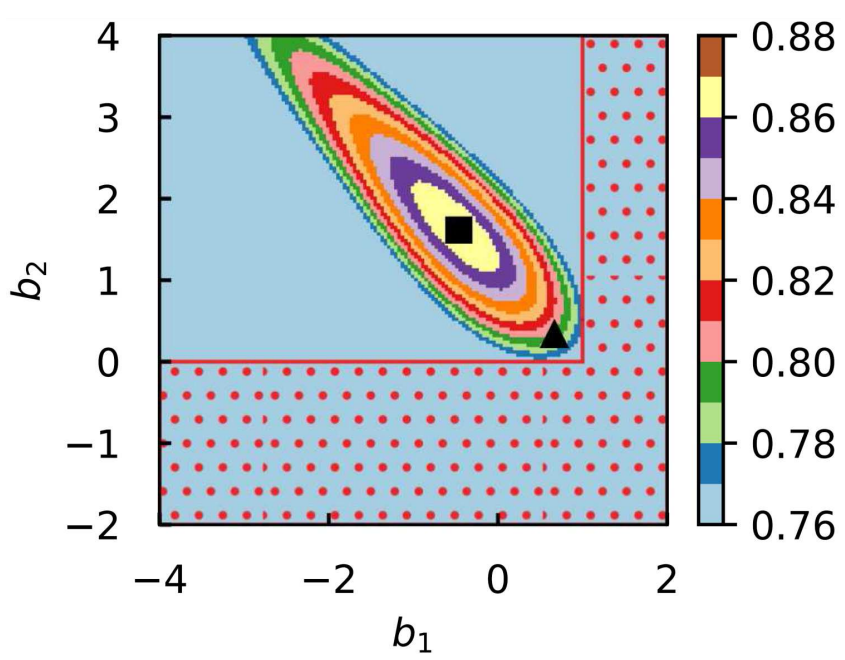

Fig. 9. Landscape of the target fidelity in a parameter space for $T=1$ for rectangular pulsed control (12) with $K=2$. Black triangle corresponds to a simple "quasilinear" control $\left(b_{1}=2 / 3\right.$ and $\left.b_{2}=1 / 3\right)$, and rectangular corresponds to the maximum output fidelity.

\subsection{Chain stitching with pulsed control}

We can investigate the opposite problem: stitching of a spin chain, i.e. transformation from the ground state of an open chain to the ground state of a closed one. In this case we can rewrite the restrictions (9) in the following form:

$$
\left.\frac{\mathrm{d} g(t)}{\mathrm{d} t}\right|_{t=0}>0,\left.\quad \frac{\mathrm{d} g(t)}{\mathrm{d} t}\right|_{t=T}>0 .
$$

For stitching under the pulsed control (12) we have $b_{1}>0$ and $b_{K}<1$. In Table I we put numerical results for cutting and stitching processes for pulse control with $K=2$. As can be seen, the optimal values of $b_{1}$ and $b_{2}$ satisfy conditions (14) imposed on them.

TABLE I

Non-optimized and optimized fidelity for pulse controlled cutting and stitching spin chain with $N=5, B=0.5$, $J=1$.

\begin{tabular}{c|c|c|c|c}
\hline \hline & $T=1$ & $T=2$ & $T=3$ & $T=4$ \\
\hline \multicolumn{5}{c}{ Cutting the spin chain } \\
\hline$f_{T 0}$ & 0.80 & 0.88 & 0.95 & 0.99 \\
$f_{T}$ & 0.87 & 0.92 & 0.97 & 0.99 \\
$b_{1}$ & -0.48 & 0.37 & 0.58 & 0.68 \\
$b_{2}$ & 1.59 & 0.70 & 0.49 & 0.45 \\
\hline \multicolumn{5}{c}{ Stitching the spin chain } \\
\hline$f_{T 0}$ & 0.80 & 0.88 & 0.95 & 0.99 \\
$f_{T}$ & 0.87 & 0.92 & 0.97 & 0.99 \\
$b_{1}$ & 1.59 & 0.70 & 0.49 & 0.45 \\
$b_{2}$ & -0.48 & 0.37 & 0.58 & 0.68
\end{tabular}

\section{Conclusions}

Despite the difficulties in analytical investigation of the time-ordered propagator (4) we have found general restrictions (9) on the shape of efficient optimized control functions. These conditions follow from the assumption of existence of a continuous transformation from a nonadiabatic to the adiabatic time domain. This transformation is possible when the lowest energy levels of the Hamiltonian do not cross (7). Thus, we connect the behavior of a complex quantum system in adiabatic and non-adiabatic time domains. Although our proposal is based on the locality of the applied control, our conclusions (9) are not limited either by the locality or by a special kind of a quantum system such as a spin chain. This analysis can be applied for speeding up transformation of any complex quantum system which can be transformed in the adiabatic way, including optical control of cold atoms [17].

\section{Acknowledgments}

We gratefully acknowledge National Research, Development and Innovation Office of Hungary (Project Nos. K124351 and 2017-1.2.1-NKP-2017-00001), Basque Government (Grant No. IT472-10), the Spanish Ministry 
of Economy, Industry, and Competitiveness (MINECO) and the European Regional Development Fund FEDER Grant No. FIS2015-67161-P (MINECO/FEDER, UE).

\section{References}

[1] M.A. Nielsen, I. Chuang, Quantum Computation and Quantum Information, Cambridge University Press, 2000.

[2] M. Busl, G. Granger, L. Gaudreau, R. Sánchez, A. Kam, M. Pioro-Ladrière, S.A. Studenikin, P. Zawadzki, Z.R. Wasilewski, A.S. Sachrajda, G. Platero, Nature Nanotechnol. 8, 261 (2013).

[3] M. Russ, G. Burkard, J. Phys. Condens. Matter 29, 393001 (2017).

[4] P.V. Pyshkin, E.Y. Sherman, J.Q. You, L.A. Wu, New J. Phys. 20, 105006 (2018).

[5] A. Das, B.K. Chakrabarti, Rev. Mod. Phys. 80, 1061 (2008).

[6] E. Farhi, J. Goldstone, S. Gutmann, M. Sipser, arXiv:quant-ph/0001106 (2000).

[7] M. Pino, J.J. Garca-Ripoll, New J. Phys. 20, 113027 (2018).
[8] M. Born, V. Fock, Z. Phys. 51, 165 (1928).

[9] M. Demirplak, S.A. Rice, J. Phys. Chem. A 107, 9937 (2003).

[10] M.V. Berry, J. Phys. A Math. Theor. 42, 365303 (2009).

[11] J. Jing, L.A. Wu, T. Yu, J.Q. You, Z.M. Wang, L. Garcia, Phys. Rev. A 89, 032110 (2014).

[12] P.V. Pyshkin, D.W. Luo, J. Jing, J.Q. You, L.A. Wu, Sci. Rep. 6, 37781 (2016).

[13] B. Damski, J. Statist. Mech. Theory Exp. 2014, P12019 (2014).

[14] E. Torrontegui, S. Ibáñez, S. Martínez-Garaot, M. Modugno, A. del Campo, D. Guéry-Odelin, A. Ruschhaupt, X. Chen, J.G. Muga, in: Adv. At. Mol. Opt. Phys., Elsevier, 2013, p. 117.

[15] J.J. Sakurai, J.J. Napolitano, Modern Quantum Mechanics, 2nd ed., Pearson, 2010.

[16] R. Fletcher, Practical Methods of Optimization, 2nd ed., Wiley, 1988.

[17] A. Alberti, unpublished results. 